\title{
A Novel KPI for Continuously Monitored Safety Barriers based on Probabilistic Safety Margins
}

\author{
F. DI MAIO ${ }^{1}$, O. SCAPINELLO ${ }^{1}$, E. ZIO ${ }^{1,2,3}$, C. CIARAPICA ${ }^{4}$, L. DECARLI ${ }^{4}$, \\ L. LA ROSA ${ }^{4}$ \\ ${ }^{1}$ Energy Department, Politecnico di Milano, Via La Masa 34, 20156 Milano, Italy, \\ francesco.dimaio@polimi.it,oscarscapinello@gmail.com,enrico.zio@polimi.it \\ ${ }^{2}$ Mines ParisTech, PSL Research University, Sophia Antipolis, France \\ ${ }^{3}$ Eminent Scholar, Department of Nuclear Engineering, Kyung Hee University, Republic of Korea \\ ${ }^{4}$ Eni E\&P division, via Emilia 1, 20097 San Donato Milanese, Italy, \\ costanza.ciarapicaalunni@eni.com,luca.decarli@eni.com,laura.larosa@eni.com
}

\begin{abstract}
In oil and gas upstream plants, several barriers (technical, procedural and organizational) are in place to prevent and mitigate accidents. Proper safety barriers functionality is, then, important to control the risk during the life of the plants. Safety barriers modelling is, then, required for risk assessment. In this work, we model the barriers functionality by discrete Health States (HSs) and their stochastic process of transition by a multistate Bayesian Network (BN). For each barrier, the HS is defined with reference to properly defined Key Performance Indicators (KPIs). Here, for technical barriers that can be continuously monitored, we propose a specific KPI based on Probabilistic Safety Margins (PSMs). Its application is illustrated with respect to the Process Control System (PCS) of the slug catcher of the upstream plant, which continuously controls the process pressure of the system within a specific operational range.
\end{abstract}

Keywords: Risk Assessment, Safety Barriers, Multistate Variables, Bayesian Networks, Continuous Monitoring, Key Performance Indicators, Probabilistic Safety Margins, Process Control System, Slug Catcher.

\section{Introduction}

In the oil and gas industry, safety by design is pursued with the implementation of preventive and mitigative safety barriers, for reducing the probability of accident initiating events and mitigating the consequences, respectively (ISO 17776). The performance of safety barriers has been assessed resorting to simplified risk indexes (Cozzani et al., 2009), Monte Carlo simulation (Abdolhamidzadeh et al., 2010) and Bow-Tie (BT) diagrams (Cherubin et al., 2011); in all these cases, the influence of the safety barriers current health state on their performance has been neglected (Landucci et al., 2016).

In the accompanying paper (Di Maio et al., 2020a), a multistate Bayesian Network (BN) has been proposed to consider the contribution of the safety barrier health state on the risk assessment. Risk measures are periodically updated based on the Knowledge, Information and Data (KID) (Zio, 2016) that can be gathered from different sources, e.g., by monitoring system and component parameters, collecting field data, reporting inspection and maintenance information, etc. In such BN framework, the safety barriers are described by their current Health State (HS) and their Failure Probability (FP). The probability $P($.
) of the barrier performance in a given HS (i.e., High (H), Medium (M), Low (L)) is estimated on the basis of specific Key Performance Indicators (KPIs) related to field data collected at the plant for a specific time, whereas the barrier FP is estimated resorting to different techniques, depending on the barrier type (i.e., technical, procedural, organizational).

In this work, we use the concept of Probabilistic Safety Margins (PSM) (Zio et al., 2010; Di Maio et al., 2016) to define a KPI for technical safety barriers that are continuously monitored. The specific case of the preventive barriers of the slug catcher of the upstream plant is considered.

The remainder of the paper is arranged as follows: Section 2 presents the case study, Section 3 the BN used for the risk assessment, Section 4 focuses on the procedure for calculating the PSM and Section 5 shows the use as KPI within the multistate BN modelling framework of Section 3; finally, in Section 6 some conclusions are drawn.

\section{Case Study}

The case study is the same as that of the companion paper (Di Maio et al., 2020a) and regards the slug catcher unit of the onshore upstream plant. The slug catcher receives the

Proceedings of the 30th European Safety and Reliability Conference and the 15th Probabilistic Safety Assessment and Management Conference 
incoming multiphase flow from the offshore platforms and performs a preliminary gas liquid separation, thus representing the first process bottleneck of the onshore facility. Several hazards (e.g., human error, equipment failure and deviation from operating conditions) could lead to the Loss of Primary Containment (LOPC) of the slug catcher, from which different consequences, namely FF, JF, PF and EX, can arise. Some preventive safety barriers (e.g., Process Safety Management System (PSMS), Task Management (TM), Design Integrity (DI), Operating Integrity (OI), Process Control (PC) and Pressure Protection System (PPS)) are considered to reduce the LOPC probability, and some recovery barriers (e.g., Isolation \& Depressurization (I\&D), Fire Management (FM), Emergency Response System (ERS) and Spill Containment System (SCS)) are installed to mitigate the effects of the accident consequences.

\section{The multistate BN}

The BN shown in Figure 1 is used to model the system and its safety barriers (see the companion paper (Di Maio et al., 2020a) for further details). The pivot node "Slug catcher LOPC", fed by the preventive safety barriers nodes, leads to the intermediate nodes describing the ignition escalation, fed by the recovery barriers, and ultimately to the consequence node. In the BN, then, each safety barrier corresponds to a node, each one characterized by Health States (HS) and the corresponding Failure Probability (FP). Different approaches can be adopted for evaluating HSs and FPs of different types of barriers and with the different types data available (see also the accompanying papers (Di Maio et al., 2020a) and (Di Maio et al., 2020c) for further details).
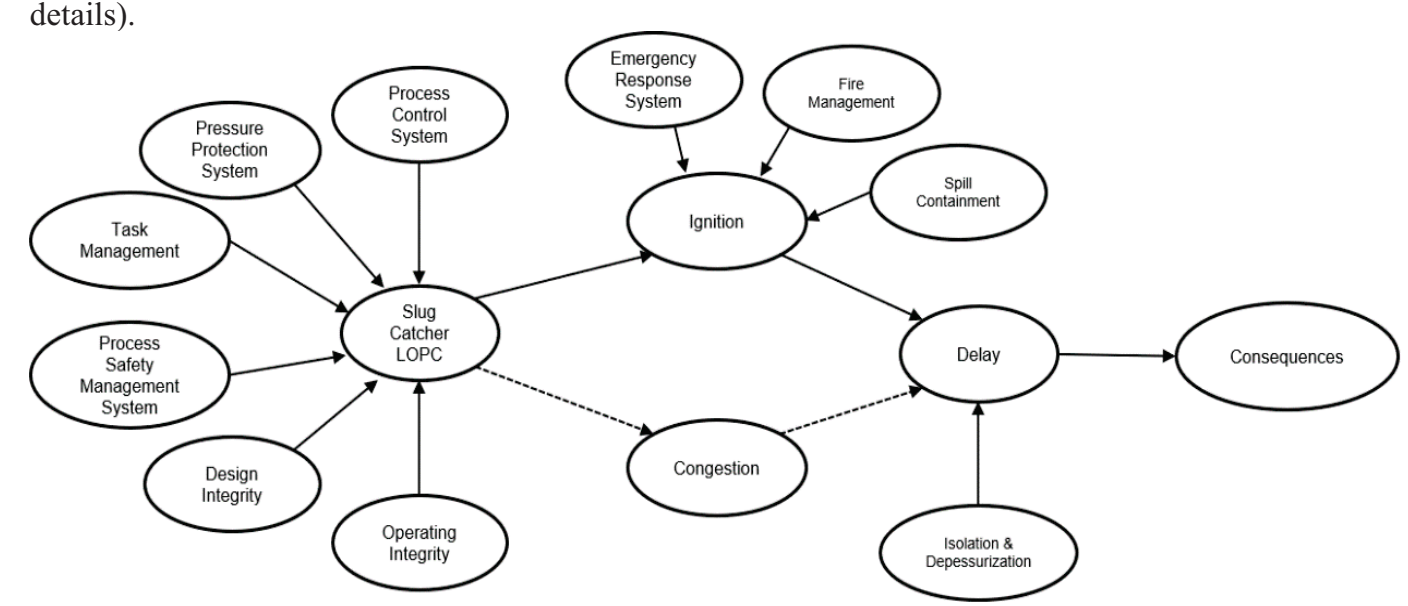

Once the barriers have been fully characterized in terms of the values of HS and FP, the CPTs of the $\mathrm{BN}$ can be filled and the probability of the consequences, $P_{\text {Cons }}\left({ }^{\circ}\right)$, can be evaluated by the $\mathrm{BN}$ rules.

\subsection{HS evaluation}

The HS reflects the health condition of the barrier, which is defined as High (H), Medium (M) and Low (L), corresponding to optimal, acceptable and poor performances respectively. The probability that the HS of a barrier is $\mathrm{H}, \mathrm{M}$ or $\mathrm{L}$ is quantified on the basis of specific Key Performance Indicators (KPIs), for each barrier. To model the uncertain relationship between KPIs and HS $\{H, M, L\}$ we resort to a probabilistic relationship, where prototypical conditions are used as anchor points. Anchors provide the analysts with examples for which the assessment is somewhat "natural" and which can be used for assessing, by comparison, situations which deviate from anchors, thus reducing subjectivity. In line with (Zio et al., 2009), a KPI is described by continuous input variables $X_{K P I}$ with values $x_{K P I}$ in the range $[0,1]$. For a specific KPI value $x_{K P I}$ of $X_{K P I}$, there can be uncertainty with respect to the HS $\{\mathrm{H}, \mathrm{M}, \mathrm{L}\}$ of the barrier, though, typically, the higher the $x_{K P I}$, the better the overall barrier performance. The HS of a safety barrier is, then, represented by a random variable $X_{H S}$ whose possible states are $\{\mathrm{H}, \mathrm{M}, \mathrm{L}\}$. The probabilities $P\left(x_{H S} \mid x_{K P I}\right)$ of being in state $x_{H S}$ given the assessed KPI value $x_{K P I}$ are obtained with the anchor points listed in Table 1 and result in the distributions shown in Figure 2. Depending on the kind of information available for the barrier of interest, several KPIs can be defined.

Fig. 1. BN of the slug catcher LOPC. 
Table 1. Anchor points.

\begin{tabular}{ccc}
\hline$x_{H S}$ & $x_{K P I}$ & $P\left(x_{H S} \mid x_{K P I}\right)$ \\
\hline $\mathrm{H}$ & 0 & 0.01 \\
& 0.4 & 0.01 \\
& 1 & 0.89 \\
\hline $\mathrm{M}$ & 0 & 0.10 \\
& 1 & 0.10 \\
\hline $\mathrm{L}$ & 0 & 0.89 \\
& 0.7 & 0.01 \\
& 1 & 0.01 \\
\hline
\end{tabular}

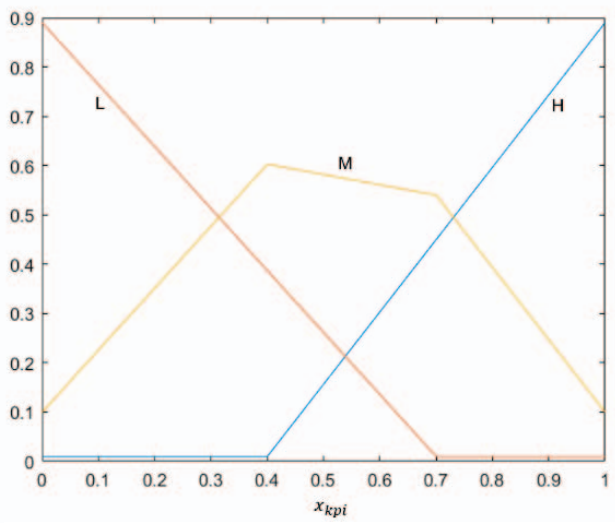

Fig. 2. Probability distributions $P\left(x_{H S} \mid x_{K P I}\right)$ for the HSs H, $\mathrm{M}$ and $\mathrm{L}$.

\section{Probabilistic Safety Margins (PSM) as KPI}

In this Section, we show the procedure for calculating the PSMs and their use as KPI for evaluating $P(\cdot)$ of a continuously monitored technical barrier. Let us define $\bar{y}(t)=$ $\left[y\left(t_{1}\right), y\left(t_{2}\right), \ldots, y\left(t_{n}\right)\right]$ as the data series taken by sensors on monitored barriers at the discrete times $t_{1}<t_{2}<\cdots<t_{n}$. PSMs can be used to measure, in probabilistic terms, to which extent the safety parameter $y$ is below an upper threshold value $U$ (or above a lower value $L$ ) during a monitoring interval. This is done by calculating the estimate $\hat{y}_{\gamma}$ of the true $\gamma$-percentile (usually the 95th percentile) of the distribution of the safety parameter, accounting for both the aleatory and epistemic uncertainties (Di Maio et al., 2016) with confidence $\beta$ (usually $95 \%$ ), such that:

$$
\begin{aligned}
& \gamma=P\left\{y<y_{\gamma}\right\} \\
& \beta=P\left\{y_{\gamma}<\hat{y}_{\gamma}\right\}
\end{aligned}
$$

The estimate $\hat{y}_{\gamma}$ indicates to which extent the barrier has been operating during the monitoring interval in normal (i.e., $\mathrm{HS}=\{\mathrm{H}\}$ ) or degraded (i.e., $\mathrm{HS}=\{\mathrm{M}, \mathrm{L}\})$ conditions. Its estimation can be done by empirical percentile estimation methods (in case of abundance of monitoring data) or by Order Statistics (OS) (Nutt \& Wallis. 2004) that provides the minimum number $S$ of samples to properly estimate $\hat{y}_{\gamma}$, with the desired confidence $\beta$. Without loss of generality, considering the safety parameter $y$ to be limited from above by the threshold $U$ (the extension to $L$ being straightforward), the approach shows that the $m$ th member $y_{m}$ of the $S$ samples sorted by descending value has a certain probability $\beta$ of exceeding the unknown true $\gamma$-th percentile $y_{\gamma}$. Then, one has a level of confidence $\beta$ that the actual value of $y_{\gamma}$ is less than the value obtained for $y_{m}$ : if $y_{m}$ meets the criterion of being less than the safety threshold $U$, then the unknown $y_{\gamma}$ will do so, too.

For an upper threshold $U$, the PSM can be calculated as:

$$
P S M= \begin{cases}\frac{U-\hat{y}_{\gamma}}{U-y_{r e f}} & \text { if } \hat{y}_{\gamma} \leq U \\ 0 & \text { if } \hat{y}_{\gamma}>U \\ 1 & \text { if } \hat{y}_{\gamma}<y_{r e f}\end{cases}
$$

and for the lower threshold $L$, as:

$$
P S M= \begin{cases}\frac{\hat{y}_{\gamma}-L}{y_{\text {ref }}-L} & \text { if } \hat{y}_{\gamma} \geq L \\ 0 & \text { if } \hat{y}_{\gamma}<L \\ 1 & \text { if } \hat{y}_{\gamma}>y_{r e f}\end{cases}
$$

where $y_{\text {ref }}$ is a reference value for $y$, which can also be considered as the nominal value of the safety parameter.

The PSM thus evaluated is, then, taken as $x_{K P I}$ for the evaluation of the probability of monitored technical barriers to be in $\mathrm{HS}\{\mathrm{H}, \mathrm{M}, \mathrm{L}\}$.

\section{Application}

We consider the Process Control System (PCS) of the slug catcher unit of the onshore upstream presented in Section 2. The PCS of the slug catcher is a monitored safety barrier that keeps the system pressure within the operational range, as close as possible to the nominal pressure value. As seen in Section 4, PSMs can be defined as a KPI for HS evaluation. For explanatory purposes, let us consider the time series $\bar{y}(t)$ plotted in Figure 4, consisting in $n=1770$ data collected over a monitoring time equal to one year. 


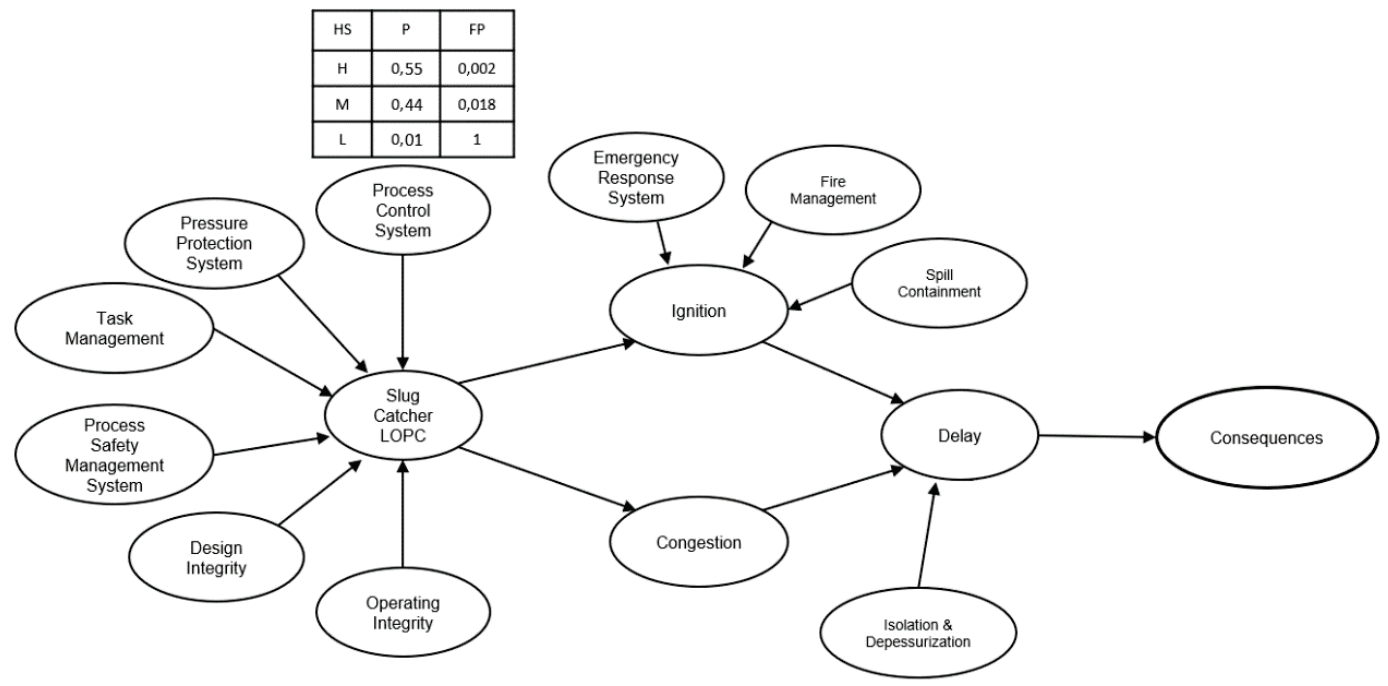

Fig. 3. BN with the CPT of the PCS

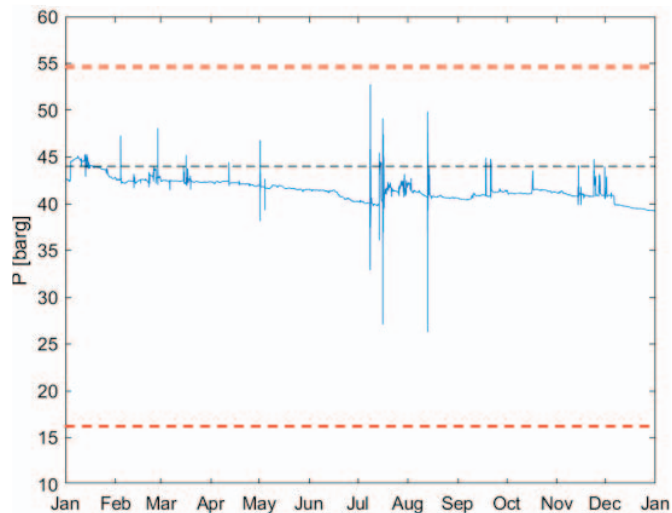

Fig. 4. Pressure evolution of the slug catcher in one-year operation. Dotted lines show nominal pressure (black), upper and lower thresholds (red).

The monitored slug catcher works at a nominal pressure set at 44 barg. Thresholds are set with respect to the design pressure of the slug catcher: the upper threshold $(U)$ at 54 barg; the lower threshold $(L)$ at 16 barg. Figure 5 shows the same time series $\bar{y}(t)$ of Figure 4 filtering off outliers (namely, data points distant more than 1.5 interquartile ranges above the third quartile or below the first quartile of the pressure distribution), since they have are not significant to indicate the HS of the PCS barrier.

Resorting to OS, for $\gamma=95 \%, \beta=95 \%$ and $m=1$ (see Section 3), the PSM for the lower $\left(\mathrm{PSM}_{\mathrm{L}}\right)$ and the upper $\left(\mathrm{PSM}_{\mathrm{U}}\right)$ thresholds are: $\mathrm{PSM}_{\mathrm{L}}=0.771$ and $\mathrm{PSM}_{\mathrm{U}}=0.840$. To be conservative, the lowest PSM is used to evaluate the HS probabilities, leading to $P_{P C S}(H)=0.55, P_{P C S}(M)=0.44$ and $P_{P C S}(L)=0.01$. These values are introduced into

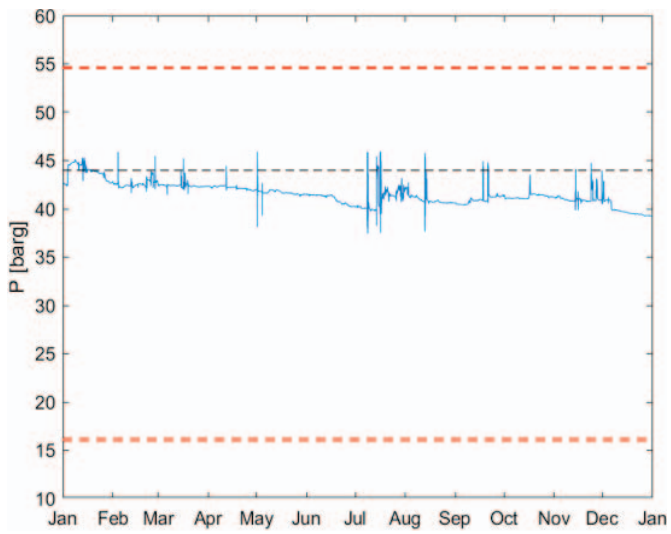

Fig. 5. Pressure evolution of the slug catcher in one-year operation, without outlier values. Dotted lines show nominal pressure (black), upper and lower thresholds (red).

the $\mathrm{BN}$ in order to fill the CPT of the PCS, as shown in Figure 3. Figure 6 shows the complete $\mathrm{BN}$ for the risk assessment of the system, where the CPTs of the preventive safety barriers (including the PCS just characterized) are in place and their effect on the consequence probability is considered (see the companion paper (Di Maio et al., 2020a) for further details). Assuming that the safety barrier is monitored for the following year as well, collecting an additional time series of data (plotted in Figure 7), we are able to compute a new $\mathrm{PSM}_{U}$ and a new $\mathrm{PSM}_{\mathrm{L}}$. With a new set of PSMs, it becomes possible to evaluate updated HS probabilities for the PCS. Thus, the updated PSMs are: $\mathrm{PSM}_{U}=0.972$ and $\mathrm{PSM}_{\mathrm{L}}=0.518$. Again, the lowest PSM is used to evaluate the following HS probabilities: $P_{P C S}(H)=0.18, P_{P C S}(M)=$ 0.58 and $P_{P C S}(L)=0.24$. As the gap between the 


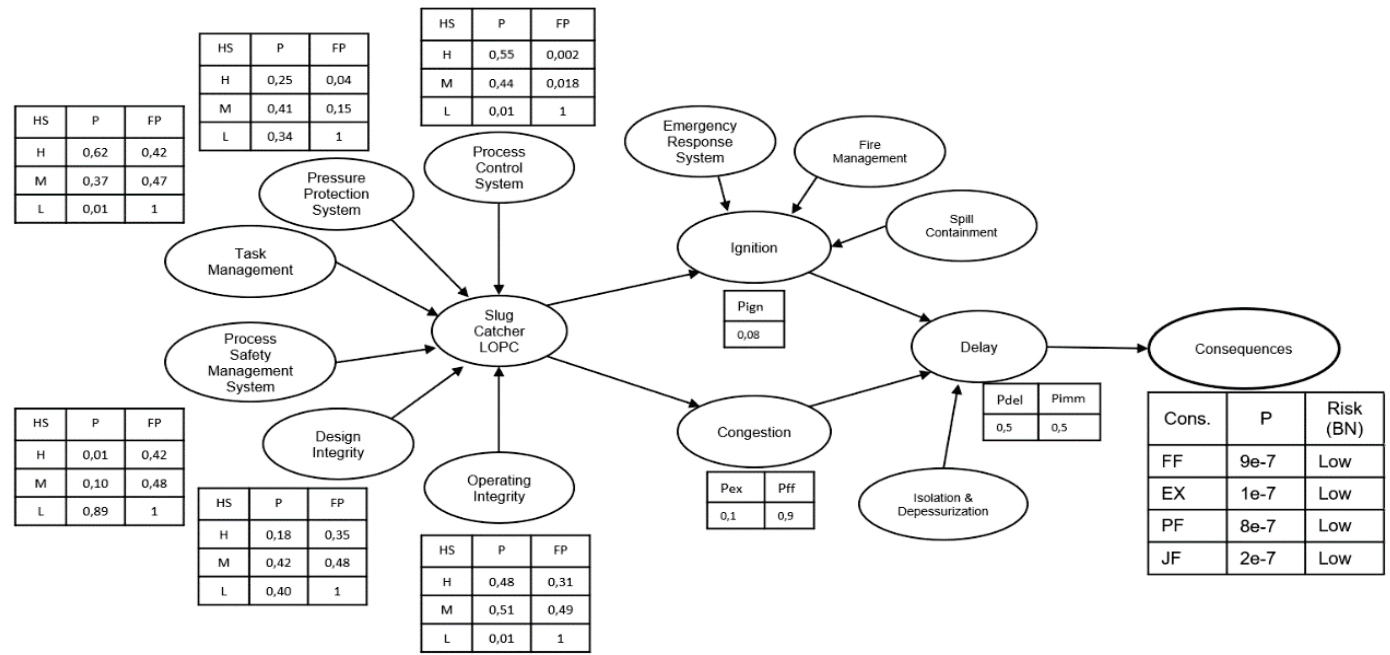

Fig. 6. BN complete with safety barriers CPTs

monitored pressure and the nominal pressure has widened, it is possible that the PCS performance has deteriorated, which is reflected by the increased probabilities of a medium or low HS and the complementary decreased probability of a high HS. The updated CPT is shown in the BN of Figure 8. The updated $\mathrm{BN}$ for the consequence risk assessment, considering the new CPT for the PCS and the remaining preventive barrier affecting the system, is shown in Figure 9.

\section{Conclusions}

This work has developed a novel KPI for the evaluation of the health conditions and performances of a technical safety barrier which is continuously monitored by sensors. Said KPI has been evaluated resorting to PSMs, a powerful tool allowing to account for uncertainties within defined confidence intervals. By monitoring

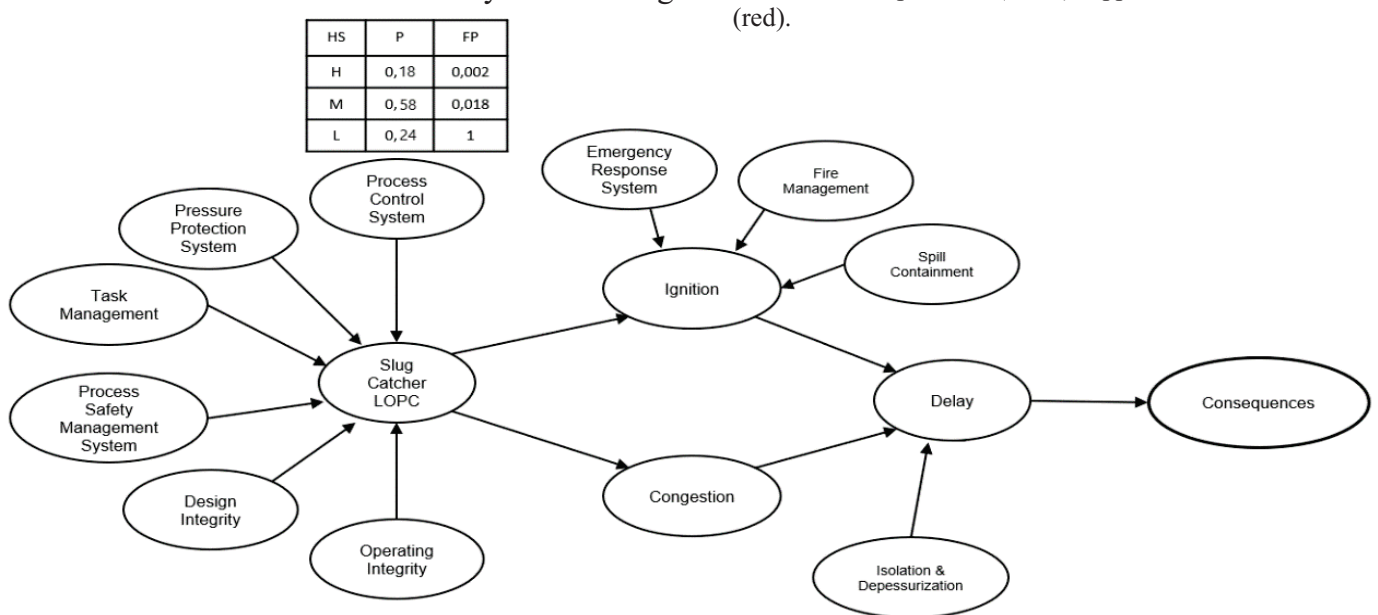

Fig. 8. BN with the updated CPT of the PCS 


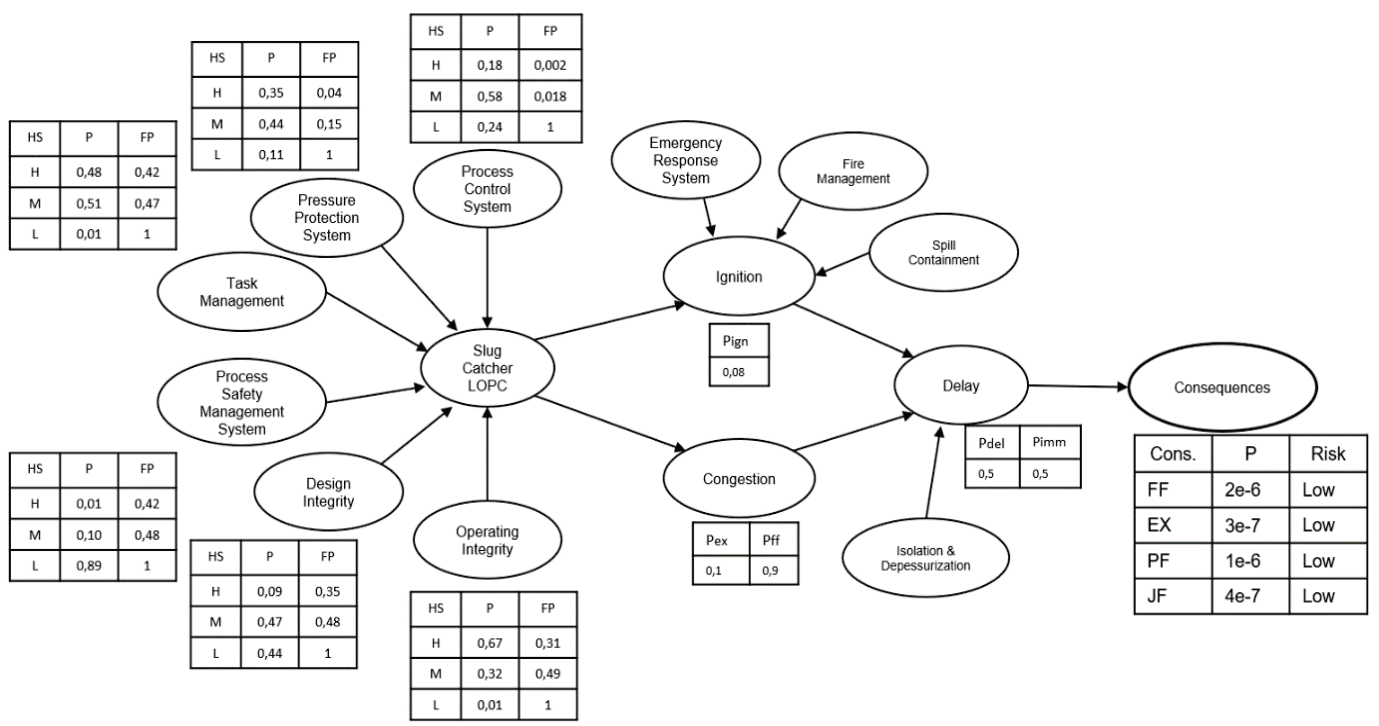

Fig. 9. Updated BN of the slug catcher LOPC.

safety variables, we are able to evaluate PSMs with respect to critical safety thresholds of a monitored barrier. PSMs are therefore an ideal KPI to evaluate barrier performance and condition. The application of the proposed methodology, shown in the case study, has led to the characterization of a PCS barrier based on its actual ability to control its characteristic safety variable (i.e., pressure). Furthermore, with the help of the BN modelling framework, the novel KPI has been updated incorporating new data, providing up to date HS evaluation and risk assessment, which is hardly done in current practice. The ability to produce updated evaluations of barrier conditions and performances paves the way to dynamic risk assessment.

\section{References}

Abdolhamidzadeh, B., Abbasi, T., Rashtchian, D., Abbasi, S.A. (2010). A new method for assessing domino effect in chemical process industry. J. Hazard. Mater. 182.

Abimbola, M., Khan, F., Khakzad, N., Butt, S. (2015). Safety and risk analysis of managed pressure drilling operation using Bayesian network. Saf. Sci. 76, 133-144.

Cherubin, P., Pellino, S., \& Petrone, A. (2011). Baseline risk assessment tool: A comprehensive risk management tool for process safety. Process Safety Progress, 30(3), 251-260.

Cozzani, V., Tugnoli, A., Salzano, E. (2009). The development of an inherent safety approach to the prevention of domino accidents. Accid. Anal. Prev. 41.
Di Maio, F., Rai, A., \& Zio, E. (2016). A dynamic probabilistic safety margin characterization approach in support of Integrated Deterministic and Probabilistic Safety Analysis. Reliability Engineering \& System Safety, 145, 9-18.

Di Maio, F., Scapinello, O., Zio, E., Ciarapica Alunni, C., Decarli, L., La Rosa, L. (2020a). A Multistate Bayesian Network for accounting the degradation of safety Barriers in the Living Risk Assessment of Oil and Gas Plants. Proceedings of the ESREL2020 PSAM15 Conference, 21-26 June 2020, Venice, Italy.

Di Maio, F., Scapinello, O., Zio, E., Ciarapica Alunni, C., Decarli, L., La Rosa, L. (2020c). Analytic Hierarchy Process for the Estimation of the Probability of Failures of Safety Barriers in Oil and Gas Installations. Proceedings of the ESREL2020 PSAM15 Conference, 21-26 June 2020, Venice, Italy.

ISO 17776 (2016), Petroleum and natural gas industries - Offshore production installations - Major accident hazard management during the design of new installations.

Landucci, G., Argenti, F., Spadoni, G., \& Cozzani, V. (2016). Domino effect frequency assessment: The role of safety barriers. Journal of Loss Prevention in the Process Industries, 44, 706-717.

Zio, Enrico. (1996). On the use of the analytic hierarchy process in the aggregation of expert judgments. Reliability Engineering \& System Safety. 53. 127138.

Zio, E., Podofillini, L., Baraldi, P., Dang, V., Librizzi, M., \& Conti, M. (2009). A Bayesian network model for dependence assessment in human reliability analysis. Reliability, Risk, and Safety. 
Proceedings of the 30th European Safety and Reliability Conference and the 15th Probabilistic Safety Assessment and Management Conference

Zio, E., Di Maio, F., \& Tong, J. (2010). Safety margins confidence estimation for a passive residual heat removal system. Reliability Engineering \& System Safety, 95(8), 828-836.

Zio, Enrico (2016). Some Challenges and Opportunities in Reliability Engineering. IEEE Transactions on Reliability, 65(4), 1769-1782. 